\title{
Comparison of residential and nursing home care before and after the 1993 community care policy
}

\author{
Vivienne L S Crawford, T R O Beringer, R W Stout
}

Department of Geriatric Medicine Queen's University of Belfast, Whitla Medical Building, Belfast BT9 7BL

Vivienne L S Crawford,

lecturer

R W Stout,

professor

Department of Health Care for the Elderly, Royal

Victoria Hospital, Belfast BT12 6BA

T R O Beringer, consultant physician

Correspondence to: Dr Crawford belfast.ac.uk

BMJ 1999;318:366 v.crawford@queens-

The NHS and Community Care Act 1990 moved responsibility for supplementing those in residential and nursing home care from social security to local authorities and was accompanied by a transfer of funds. ${ }^{1}$ This system was implemented on 1 April 1993 with the aim that care should be provided and funded in accordance with the person's need for care.

For the first time the level of dependency and need would influence financial support for admission to an institutional care facility. If the aim was achieved and care is now better targeted a clearer differentiation in levels of dependency between residential and nursing care would be expected after 1 April 1993, with a higher level of dependency in nursing homes.

\section{Subjects, methods, and results}

To test whether the new policy has resulted in better targeting of care we examined physical dependency and mental state of all residents aged over 65 years admitted to residential and nursing care in Belfast from 1 April 1992 to 31 March 1993 and 1 April 1993 to 31 March 1994-that is, 1 year before and 1 year after implementation of the policy-by using several standard scoring systems (Barthel index²; survey version of Clifton assessment procedure for the elderly (CAPE), ${ }^{3}$ which includes a measure of physical dependency and information or orientation which are combined to give a CAPE score; abbreviated mental score; ${ }^{4}$ and the behavioural rating score of the CAPE). The data from each measure of dependency were subjected to analysis of variance followed by multiple regression analysis to assess the impact of the community care policy. The analysis of variance highlighted significant factors (type of care, sex, and period of admission) and two way interactions which were then entered into a multiple regression analysis with a full factorial design. The effect of each factor and interaction on each measure of dependency relative to a reference group (residential care, male sex, before April 1993) were thus determined. (The effect for a non-reference group was calculated from $\beta$ and its significance from a " $\mathrm{t}$ " value.)

Mean (SE) scores for each measure of dependency by type of care and period of admission. Increasing dependency is indicated by increase in physical dependency or behavioural rating score or decrease in abbreviated mental score, Barthel index, information or orientation score, or Clifton assessment procedure for the elderly (CAPE) score

\begin{tabular}{|c|c|c|c|c|}
\hline \multirow[b]{2}{*}{ Mean (SE) } & \multicolumn{2}{|c|}{ Residential care } & \multicolumn{2}{|c|}{ Nursing care } \\
\hline & $\begin{array}{l}\text { Before } 1 \text { April } \\
1993(\mathrm{n}=93)\end{array}$ & $\begin{array}{l}\text { After } 1 \text { April } \\
1993(n=123)\end{array}$ & $\begin{array}{l}\text { Before } 1 \text { April } \\
1993(n=274)\end{array}$ & $\begin{array}{l}\text { After } 1 \text { April } \\
1993(n=266)\end{array}$ \\
\hline Barthel score & $14.20(0.51)$ & $15.81(0.31)^{*}$ & $10.30(0.35)$ & $9.26(0.34) \dagger$ \\
\hline Behavioural rating score & $10.66(0.61)$ & $9.97(0.43)$ & $14.10(0.41)$ & $15.46(0.36)$ \\
\hline Abbreviated mental score & $6.22(0.39)$ & $5.78(0.33)$ & $5.29(0.23)$ & $5.15(0.23)$ \\
\hline $\begin{array}{l}\text { CAPE score (information or orientation } \\
\text { minus physical dependency) }\end{array}$ & $3.64(0.65)$ & $3.80(0.54)$ & $1.44(0.39)$ & $0.53(0.39)$ \\
\hline Information/orientation score (CAPE) & $7.72(0.42)$ & $7.41(0.35)$ & $6.81(0.25)$ & $6.48(0.25)$ \\
\hline Physical dependency score (CAPE) & $4.32(0.27)$ & $3.71(0.22)$ & $5.77(0.17)$ & $6.31(0.16)^{\star}$ \\
\hline
\end{tabular}

P $<0.001, \uparrow P<0.05$ as determined by multiple regression analysis (see text).
A total of 756 residents (166 men, 590 women), 216 in residential (93 admitted before, 123 after 1 April 1993) and 540 in nursing home care (274 admitted before, 266 after) were assessed. After 1 April 1993 admissions to residential care increased (32\%) while admissions to nursing care declined (3\%). Age did not differ between people admitted to residential or nursing care before or after 1 April 1993 (median age 83-85 years; $F=1.705, \mathrm{P}=0.164$ ). There was a significant increase in married residents admitted to nursing care after 1 April $1993\left(\chi^{2} 12.47, \mathrm{P}<0.01\right)$. The pattern of financing did not change after 1 April 1993, with $71 \%$ of residential care residents receiving supplementation before and 74\% after 1 April 1993; the figures for residents of nursing homes were $59 \%$ and $57 \%$, respectively. Results of the multiple regression analysis showed that dependency levels in nursing homes increased significantly after 1 April 1993 (mean decrease in Barthel index of 1.44 units, $\mathrm{P}<0.05$; mean increase in physical dependency of 0.53 units, $\mathrm{P}<0.001$ ) but decreased significantly in residential homes (mean increase in Barthel index of 1.96 units, $\mathrm{P}<0.001)$. Tests of mental function were unchanged (table).

\section{Comment}

Since the introduction of the new community care policy in April 1993 admission to residential and nursing care is now better targeted to achieving more appropriate care for the individual in relation to dependency determined by an assessment of need. After the introduction of assessment more people were admitted to institutional care. This suggests that there was an unmet need before April 1993. If current patterns of long term care remain unchanged by the year 2030 expenditure in long term care alone will grow from $7.5 \%$ to nearly $11 \%$ of the gross national product, illustrating the increasing need for appropriate targeting of healthcare resources. ${ }^{5}$

We thank Dr CC Patterson for statistical advice; Mr R Hadden; research nurses Mrs Marie Mooney and Mrs Sandra Dunlop for their assessments; and the proprietors and residents of the homes.

Contributors: RWS and VLSC initiated the primary study hypothesis, obtained funding, and recruited the research staff. All authors participated in the protocol design, in monitoring the study, in discussing core ideas, and in interpreting the findings. VLSC verified and analysed the data and prepared the manuscript. TROB and RWS edited and contributed to the manuscript. RWS is guarantor of the study.

Funding: Department of Health and Social Services, Northern Ireland.

Competing interests: None declared.

Department of Health and Social Services. People first: community care in Northern Ireland for the 1990s. London: HMSO, 1990.

2 Mahoney FI, Barthel DW. Functional evaluation: Barthel index. Maryland State Med J 1965;14:61-5.

3 Patti AH. A survey version of the Clifton assessment procedure for the elderly (CAPE). Br J Clin Psychol 1981;20:173-8.

4 Oureshi KN, Hodkinson HM. Evaluation of a 10 question mental test in the institutionalised elderly. Age Ageing 1974;3:152-7.

5 Nuttall SR, Blackwood RJL, Russell BMH, Cliff JP, Cornall MJ, Cowley A, et al. Financing long term care in Great Britain. J Inst Actuaries 1994;21:1-53. (Accepted 7 August 1998) 